\title{
93. A Theorem on Flat Couples
}

\author{
By Takeshi IsHIKAwA \\ Department of Mathematics, Tokyo Metropolitan University, Tokyo
}

(Comm. by Z. Suetuna, M.J.A., July 12, 1960)

In this short note, I will prove a theorem in homological algebra and its corollary, which is well known in ideal theory in integral domains.

Throughout this note any ring is assumed to be commutative and have a unit element which acts as the identity operator on any module over the ring. We will call the pair $\left(R, R^{\prime}\right)$ of a ring $R$ and its overring $R^{\prime}$ a flat couple, if $R^{\prime} / R$ is flat as an $R$-module [7]. A ring $R$ is called semi-hereditary if every finitely generated ideal of $R$ is $R$-projective [1]. Then we have the

THEOREM. Let $R$ be a semi-hereditary ring and $R^{\prime}$ be an integral (or module finite) extension ring of $R$. Then, $\left(R, R^{\prime}\right)$ is a flat couple.

The theorem is obtained directly from the following two lemmas.

LEMMA 1. A semi-hereditary ring is integrally closed in its full ring of quotients.

Proof. Let $R$ be a semi-hereditary ring and $K$ be its full ring of quotients. Let $x$ be an element of $K$ and be integral over $R$ and

$$
x^{n}+r_{1} x^{n-1}+\cdots+r_{n}=0
$$

be an equation of integral dependence satisfied by $x$ over $R$. There exists a non-zerodivisor $r$ of $R$ such that $r x^{n-i} \in R$ for $i=0,1, \cdots$, $n-1$. Since $x^{n+1}=-\left(r_{1} x^{n}+\cdots+r_{n} x\right), r x^{n+1}$ is also in $R$. Thus we have $r x^{i} \in R$ for $i=1,2, \cdots$. Now, we consider an ideal $I$ of $R$ generated by $\left(r x^{i} ; i=1,2, \cdots\right)$. Since this ideal $I$ is finitely generated (in fact, generated by $r x, r x^{2}, \cdots, r x^{n}$ ) and $R$ is semi-hereditary, $I$ is projective and by Cartan-Eilenberg [1, VII, 3.1] there exist $R$-homomorphisms $\varphi_{i}: I \rightarrow R$ such that $y=\sum_{i=1}^{n} \varphi_{i}(y) r x^{i}$ for all $y \in I$. Thus since $r x \in I$, it follows

$$
r x=\sum_{i=1}^{n} \varphi_{i}(r x) r x^{i}=\sum_{i=1}^{n} \varphi_{i}\left(r^{2} x^{i+1}\right)=\sum_{i=1}^{n} \varphi_{i}\left(r x^{i+1}\right) r,
$$

and since $r$ is a non-zerodivisor, we have $x=\sum_{i=1}^{n} \varphi_{i}\left(r x^{i+1}\right) \in R$. This shows that $R$ is integrally closed in $K$.

Let $A$ be an $R$-module and $a$ be a non-zero element of $A$. We say that $a$ is an $R$-torsion element if $r a=0$ for some non-zerodivisor $r$ of $R$, and $A$ is called $R$-torsion-free if $A$ has no $R$-torsion element except zero.

LEMMA 2. A ring $R$ is integrally closed in its full ring of 
quotients, if and only if $R^{\prime} / R$ is torsion-free as an $R$-module for each integral (or module finite) extension ring $R^{\prime}$ of $R$.

Proof. First, we notice that any module finite extension of $R$ is integral over $R$ following M. Nagata ([5] or [6]). Let $R^{\prime}$ be an arbitrary module finite (or integral) extension of $R$ and further $R$ be integrally closed. Let $\bar{r}^{\prime}\left(r^{\prime} \in R\right)$ be an element of $R^{\prime} / R$ and assume that there exists a non-zerodivisor $s$ of $R$ such that $s \cdot \bar{r}^{\prime}=0$. Then, $s r^{\prime}$ is in $R$, hence $r^{\prime}$ is an element of the full ring of quotients of $R$, and moreover $r^{\prime}$ is integral over $R$. Thus, since $R$ is integrally closed, $r^{\prime}$ must be in $R$, that is $\bar{r}^{\prime}=0$ in $R^{\prime} / R$. This implies that $R^{\prime} / R$ is $R$-torsion-free.

Conversely, let $x=r^{\prime} / r\left(r^{\prime} \neq 0\right)$ be an element of the full ring of quotients of $R$ and be integral over $R$. Consider an integral (and module finite) extension $R[x]$ of $R$ and suppose that $R[x] / R$ is $R$ torsion-free. Then, since $r x \in R$, i.e. $r \bar{x}=0$ in $R[x] / R$ and $r$ is a non-zerodivisor of $R$, it follows $\bar{x}=0$, i.e. $x \in R$. This shows that $R$ is integrally closed.

Now, we return to the theorem. From the above two lemmas, $R^{\prime} / R$ is $R$-torsion-free and following a theorem of $\mathrm{S}$. Endo [2], which is a generalization of a theorem of A. Hattori [3], any $R$-torsionfree module over a semi-hereditary ring is $R$-flat. Thus, we obtain the result.

If $\left(R, R^{\prime}\right)$ is a flat couple, we have $\mathfrak{H} R^{\prime} \curvearrowright R=\mathfrak{A}$ for each ideal $\mathfrak{A}$ of $R$ by Serre [7, Prop. 22]. Thus we have the following corollary and we will prove it to make sure of it.

COROLlARY. Let $R$ be a semi-hereditary ring, $R^{\prime}$ be an integral (or module finite) extension of $R$ and $\mathfrak{A}$ be an ideal of $R$. Then we have

$$
\mathfrak{U} R^{\prime} \curvearrowright R=\mathfrak{A} .
$$

Proof. Let $x=\sum r_{i}^{\prime} a_{i}\left(a_{i} \in \mathfrak{N}, r_{i}^{\prime} \in R^{\prime}, x \in R\right)$ be an element of $\mathfrak{Y} R^{\prime}$ $\frown R$. Since $R^{\prime} / R$ is $R$-flat, $f: R / \mathfrak{Y}\left(\cong R \bigotimes_{R} R / \mathfrak{U}\right) \rightarrow R^{\prime} \bigotimes_{R} R / \mathfrak{U}$ is a monomorphism. Then we have $f(\bar{x})=1 \otimes \bar{x}=\sum\left(1 \otimes \overline{\left.r_{i}^{\prime} a_{i}\right)}=\sum\left(r_{i}^{\prime} \otimes_{R} \bar{a}_{i}\right)=0\right.$, this implies $\bar{x}=0$ in $R / \mathfrak{U}$ i.e. $x \in \mathfrak{A}$. Thus $\mathfrak{A} R^{\prime} \curvearrowright R \subset \mathfrak{A}$ and $\mathfrak{A} \subset \mathfrak{A} R^{\prime} \curvearrowright R$ is obvious.

REMARKs. 1. I express my hearty thanks to Prof. A. Hattori for his following attention. Lemma 2 is not a proposition for " integral", but for "closed". In fact, we can state it more generally as follows. Let $R$ be a ring and $K$ be its full ring of quotients. If an element of an overring of $R$ has a certain property $P$, which can be considered for an element of an overring of $R$, we call it a $P$-element. And if each $P$-element which is in $K$ is always contained in $R, R$ is called $P$-closed in $K$. Then, " if $R$ is $P$-closed in $K, R^{\prime} / R$ 
is $R$-torsion-free for any $P$-extension $R^{\prime}$ of $R$ ", and furthermore if $R[x]$ is a $P$-extension for any $P$-element $x$, the converse holds.

2. In the case of integral domains, a semi-hereditary ring is called a Prüfer ring. It is easily seen that a Prüfer ring is integrally closed, since an integral domain is a valuation ring if and only if it is a local Prüfer ring (cf. [4]). And a torsion-free module over a Prüfer ring is flat by A. Hattori's theorem [3].

\section{References}

[1] H. Cartan-S. Eilenberg: Homological Algebra, Princeton University (1956).

[2] S. Endo: Forthcoming.

[3] A. Hattori: On Prüfer rings, J. Math. Soc. Japan, 9 (1957).

[4] E. Matlis: Injective modules over Prüfer rings, Nagoya Math. Jour., 14 (1959).

[5] M. Nagata: Basic theorems on general commutative rings, Memoirs of the College of Science, Univ. Kyoto, series A, 29, Math., no. 1 (1955).

[6] M. Nagata: On the theory of semi-local rings, Proc. Japan Acad., 26 (1950).

[7] J. P. Serre: Géométrie algébrique et géométrie analytique, Ann. Inst. Fourier, Grenoble, 6 (1955-1956). 